\title{
Impacts of impulsive noise from partial discharges on wireless systems performance: application to MIMO precoders
}

Ghadir Madi ${ }^{1}$, Fabien Sacuto ${ }^{3}$, Baptiste Vrigneau ${ }^{1 *}$, Basile L Agba², Yannis Pousset ${ }^{1}$, Rodolphe Vauzelle ${ }^{1}$ and François Gagnon ${ }^{3}$

\begin{abstract}
To satisfy the smart grid electrical network, communication systems in high-voltage substations have to be installed in order to control equipments. Considering that those substations were not necessarily designed for adding communication networks, one of the most appropriate solutions is to use wireless sensor network (WSN). However, the high voltage transported through the station generates a strong and specific radio noise. In order to prepare for such a network, the electromagnetic environment has to be characterized and tests in laboratories have to be performed to estimate the communication performances. This paper presents a method for measuring the noise due to high voltage and more particularly the impulsive noise. In the laboratory, we generate the impulsive noise using two specimens, and we show that these laboratory measurements validate the field measurements of Pakala et al. For the two specimens, it aims to link the noise characteristics (magnitude and frequency) with the specimen parameters (power supply and geometric dimensions) to predict the environments where wireless communications can be troublesome. By using different sets of this measured noise, we show that the statistical model of Middleton Class A can be used to model the impulsive noise in high-voltage substations better than the Gaussian model. We consider a cooperative multiple-input-multiple-output (MIMO) system to achieve the wireless sensor communication. This system uses recent MIMO techniques based on precoding like max- $d_{\text {min }}$ and P-OSM precoders. The MIMO precoder-based cooperative system is a potential candidate for energy saving in WSN since energy efficiency optimization is a very important critical issue. Since MIMO precoders are with Gaussian noise assumption, we evaluate the performance of several MIMO precoders in the presence of impulsive noise using estimated parameters from the measured noise.
\end{abstract}

Keywords: $\mathrm{MIMO}$, precoders, impulsive noise, high-voltage substations modeling

\section{Introduction}

In order to save energy, electricity providers have attempted to control and monitor their grid using "smart grid" regulation [1]. It consists, for example, of knowing the needs of electric equipments in consumers' home and adapting the transformation of energy from high-voltage substation. The first step is to interact with those substations by checking equipment status using sensor network technology. It is important to keep in mind that current substations will still be operational

\footnotetext{
* Correspondence: baptiste.vrigneau@univ-poitiers.fr

${ }^{1}$ XLIM-SIC UMR CNRS 6172 Bvd., Marie et Pierre Curie, 86962 Futuroscope Cedex, France

Full list of author information is available at the end of the article
}

for the next 50 years, and because of their large area, it would be very expensive to link sensors with optical fiber or cables. Therefore, one of the most appropriate solutions is to adapt a wireless network to those substations. Since wireless networks do not use expensive signal and control cables for data transmission, they are easier to install and provide a cost-effective solution for these applications. However, there is a concern: the presence of impulsive noise or electromagnetic interference (EMI) generated by high-voltage equipments. According to Pakala measurements $[2,3]$ going from DC to 10 $\mathrm{GHz}$, the noise power near wireless communication frequencies is significant (around $-50 \mathrm{dBm}$ average power for the band 1-10 GHz). We have to distinguish two 
noises: the ambient noise that is considered Gaussian and the impulsive noise. The impulsive noise is mainly due to partial discharges which occurs within high-voltage equipment or on its surface. The impulsive noise has a highly structured form characterized by significant probabilities of large interference levels and short duration [4]. The impulsive character of the interference can drastically degrade the performance and the reliability of wireless communication systems even in case of high signal-to-noise ratios. In order to guard against unacceptable performance, the true characteristics of the noise must be taken into account. To do so, one needs an accurate model for the impulsive noise.

Statistical-physical models of EMI have been derived by Middleton with three models (class A, B, and C) including the non-Gaussian components of natural and man-made noise [5]. The models are parametric with parameters explicitly determined by the underlying physical mechanisms and are canonical, i.e., their mathematical form is independent of the physical environment. The distinction between the three models is based on the relative bandwidth of noise and receiver. Middleton models have been shown to accurately model the nonlinear phenomenon governing electromagnetic interference. These models have been widely used in electromagnetic applications and communication problems $[4,6,7]$.

As mentioned before, a wireless network seems to be a good solution in a large area where electricity substation is situated. Therefore, our research focuses on the wireless sensor communication in this environment. There has recently been a great amount of research on various MIMO techniques for wireless communication systems; more particularly, cooperative MIMO and virtual antenna array concepts have been proposed to achieve MIMO capability in WSN $[8,9]$. The goal is to reduce the energy consumption of sensors since energy efficiency optimization is a very important critical issue in system design of WSN. The results have shown that in some cases, MIMO-based cooperative systems for WSN lead to better energy optimization and smaller end-to-end delay compared to the traditional singleinput single-output (SISO) approach even after taking into account the additional circuit power, communications, and training overheads [10]. These cooperative systems were based on space-time block codes (STBC). Therefore, we consider a MIMO system to achieve the wireless sensor communication in the substation. The idea is to exploit more the performance of MIMO systems by using recent MIMO techniques based on precoding like MIMO max- $d_{\min }$ [11] and P-OSM precoders [12]. MIMO precoders require the knowledge of the channel state information (CSI) at the transmitter. The precoder exploits the CSI to improve the performance of a wireless system by optimizing a pertinent criteria. MIMO precoders improve the BER and increase the spectral efficiency of the system compared to STBC codes. Therefore, the MIMO precoder-based cooperative system is a potential candidate for more energy saving design in WSN, and we will propose many cooperative schemes for these precoders in a future work. A wireless MIMO precoder-based cooperative system will suffer from the impulsive noise in the substation. In this work, the precoder does not represent a solution against the impulsive noise, but it is rather used as a promising technique for energy-efficient data transmission in WSN. In digital subscriber line (DSL) communication, various solutions are applied to mitigate the effects of impulsive noise. In [13], a variety of error control techniques are discussed. They are focused on enhancing the Reed-Solomon code performance. The application of Turbo-Codes is also considered in [14]. Other solutions can be applied to combat the impulsive noise effects like a non-linear receiver $[4,15]$. The non-linear receiver needs the knowledge of a statistical model of the noise. All these mentioned solutions could be applied to the wireless MIMO precoder-based cooperative system to improve it, but one may first need to define a standard for this system in order to choose the appropriate solution. However, we show later that if we know the statistical model of the impulsive noise in the substation, the maximum likelihood (ML) MIMO receiver could lead to a certain improvement in performance. Therefore, before installing this type of wireless system in substations, the electromagnetic environment should be heavily studied, and tests in laboratories should be performed in order to characterize the noise and evaluate its effects on the wireless system.

In this paper, we present a method for measuring partial discharges of two specimens in the laboratory and then analyze the parameters affecting wireless communications. Results show that these laboratory measurements of partial discharges validate the field measurements in [2]. From these measurements, our goal is to validate the statistical model of Middleton Class A for the high-voltage substation. Since Middleton Class A model was derived for single-antenna systems, an extension of the model is derived for multi-antenna systems. This validation allows us to consider a statistical model for the impulsive noise of partial discharges in order to evaluate its impact on a MIMO precoder wireless transmission system in a high-voltage substation (in the presence of impulsive noise).

This article is organized as follows: Section II presents a methodology for measuring impulsive noise generated by two specimens in time domain using an antenna and a digital oscilloscope, and we study how some parameters (voltage and geometric dimensions) affect the 
impulsive noise spectrum. Section III introduces a brief overview of the Middleton Class A model and focuses on the validation of this model with the measured data of partial discharges, and the extension of Middleton Class A model for multi-antenna systems is also presented. The MIMO precoders used in simulation are presented in Section IV. Performances in terms of bit error rate (BER) of MIMO precoders are evaluated in Section V. The BER of MIMO precoders is evaluated in the presence of impulsive noise modeled by Middleton Class A and compared to the BER in the presence of Gaussian noise. Finally, we draw the conclusion in Section VI.

\section{Measurements of partial discharges}

\section{A. Measurement method}

Two specimens are used to generate partial discharges, a generator bar and a Tesla-coil [16]. For the generator bar, a copper conductor is coated with epoxy-micarta insulation with a shield covers the insulation in the middle of the bar. This specimen can be considered as an unsheathed coaxial cable: The central conductor is linked to a high-voltage source, and the shield is grounded. The Tesla-coil is a tool used to ionize plasma. It delivers a high voltage $(54 \mathrm{kV})$. The same measurement setup is used for the two specimens. A wideband antenna is linked to the TDS6124C digital oscilloscope, which is set with a sampling frequency twice larger than the maximum frequency of the antenna. For example, $2.5 \mathrm{GS} / \mathrm{s}$ sampling frequency is used when the biconical antenna $(30-300 \mathrm{MHz})$ is used, and $10 \mathrm{GS} / \mathrm{s}$ is used for the Wi-Fi antenna $(2.4 \mathrm{GHz}, 2$ $\mathrm{dBi}$ ). The step-by-step procedure for the measurements is described as follows:

- Step 1: The antenna is installed at $2 \mathrm{~m}$ approximately from the specimen. The antenna is connected to an oscilloscope, located behind a Faraday fence.

- Step 2: Before feeding current into the specimen, the electromagnetic noise of the room must be measured and recorded.

- Step 3: The fence is closed, and then the specimen is fed gradually from $1 \mathrm{kV}$ until the first discharge waveform appears on the oscilloscope.

- Step 4: The oscilloscope trigger must be set to record partial discharges as soon as they appear. (Setting the trigger above twice magnitude of the background noise is recommended).

- Step 5: Once the waveform is captured, it is recorded in a file and exported for processing.

- Step 6: The FastFrame option [17] can be used to record 50 or 100 partial discharge waveforms for statistical analysis (the FastFrame waveform is also recorded in a file).
- Step 7: Statistical analysis is done using MATLAB signal processing tool.

\section{B. Specimen parameters influence 1) Magnitude rising}

With the generator bar, it was observed in the laboratory that the voltage used to feed the specimen has an influence on the partial discharge magnitude. The phenomenon has already been studied for different highvoltage substations $[2,3,18]$. For example, the power line with the highest voltage gives the more significant corona noise according to Pakala works. So it is obvious that to obtain a more powerful partial discharge signal, we have to feed the specimen with a higher voltage which is not always possible in laboratory.

\section{2) Average frequency rising}

Considering that the distance between two electrodes under high voltage can influence the spectrum in power magnitude, it is predictable that this gap can also modify the frequency band of the partial discharge noise. Knowing that this noise spectrum covers several hundreds of $\mathrm{MHz}$ for the Tesla-coil, we determine the average frequency associated with the noise signal as:

$$
f_{\text {ave }}=\sum_{i=1}^{N} f_{i} \times \frac{S_{i}}{\sum_{j=1}^{N} S_{j}}
$$

where $S_{i}$ is the spectral magnitude of the $f_{i}$ frequency, and $N$ is the number of spectrum points. With this formula and by varying the gap, we try to demonstrate the influence of the gap on the frequencies appearing in the spectrum.

Using each partial discharge spectrum, the average frequency is calculated for different gaps $(5,3.5$, and 2.5 $\mathrm{mm})$. Figure 1 shows the gap influence on average

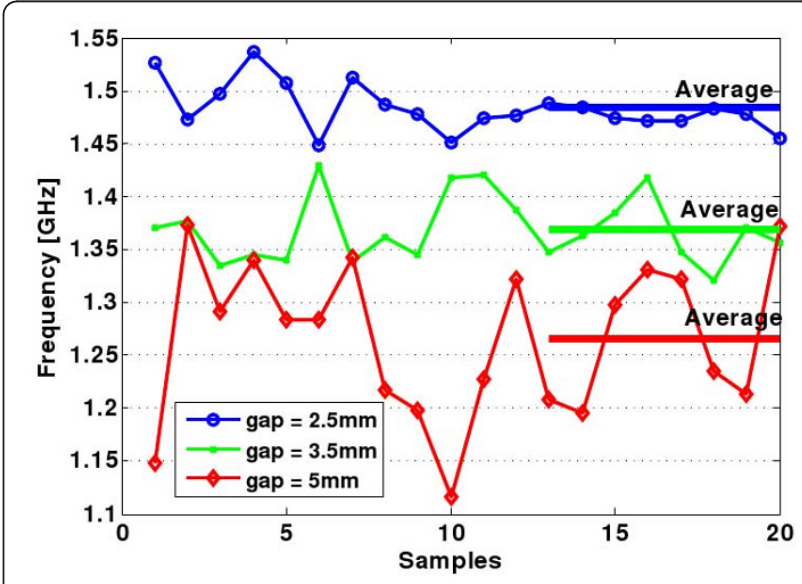

Figure 1 Average frequencies for different gaps, 54 kV power supply 
frequency for 20 partial discharges recorded (samples). Obviously, the average frequency rises when the gap gets small. From (1), it is seen that the spectrum components $\left(S_{i}\right)$ are greater for a small gap. The impulsive noise band thus moves toward the communication bands $(900 \mathrm{MHz}, 2.4 \mathrm{GHz}, 5-6 \mathrm{GHz})$ with a smaller gap size.

Considering this fact, a test bed is set up in order to observe what is the behavior of the impulsive noise spectrum around $2.4 \mathrm{GHz}$ (Figure 2). To analyze the impact of the impulsive noise to a $2.4 \mathrm{GHz}$ communication signal, a $\mathrm{Wi}-\mathrm{Fi}$ router is operating during the experiences, and its signal serves as a reference. It is placed at $14 \mathrm{~m}$ from the antenna. The signal at $2.4 \mathrm{GHz}$ is amplified about $25 \mathrm{~dB}$ by the LNA amplifier. Hence, the oscilloscope records signals around $2.4 \mathrm{GHz}$. The Wi-Fi antenna is at distance of $10 \mathrm{~m}$ from the partial discharge. The average spectrum of Figure 3 is calculated based on the recording of 100 partial discharges for different gaps in Tesla-Coil setup. From Figure 3, it is obvious that the frequencies of Tesla-Coil overlap the Wi-Fi signal, and by observing the spectrum corresponding to $2.5 \mathrm{~mm}$ gap, it is also obvious that the WiFi transmission can be significantly degraded.

Consequently, the impulsive noise can interfere with the communication bands, if the voltage is high enough and the gap small enough. Indeed, the voltage delivered by the specimens in our measurements is well below the voltage of high-voltage substations (200-700 kV). Partial discharges occur more easily in substations because the air dielectric strength can be reached by higher voltage and for bigger gap than we used $(360 \mathrm{kV}$ for a $10 \mathrm{~cm}$ gap for example).

\section{Statistical model of measurements and noise model for multi-antenna system}

In this section, we will validate the statistical model of Mid-dleton Class A with the measured data of partial discharges obtained in Section II. Here, we do not focus on the frequency of the noise. We first present a brief overview of the Middleton Class A model. Then, we focus on the validation of this model with the measured

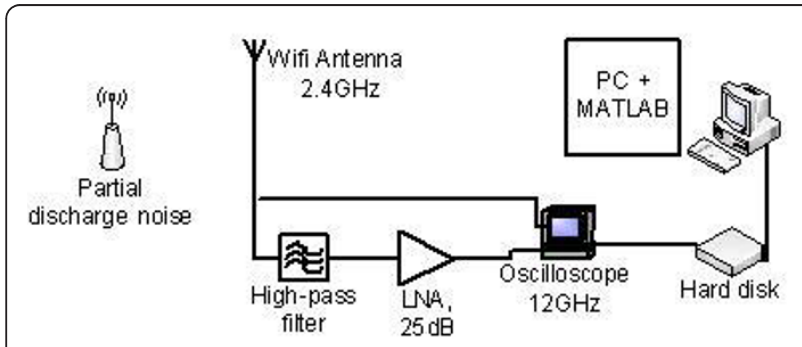

Figure 2 2.4 GHz measurement setup.

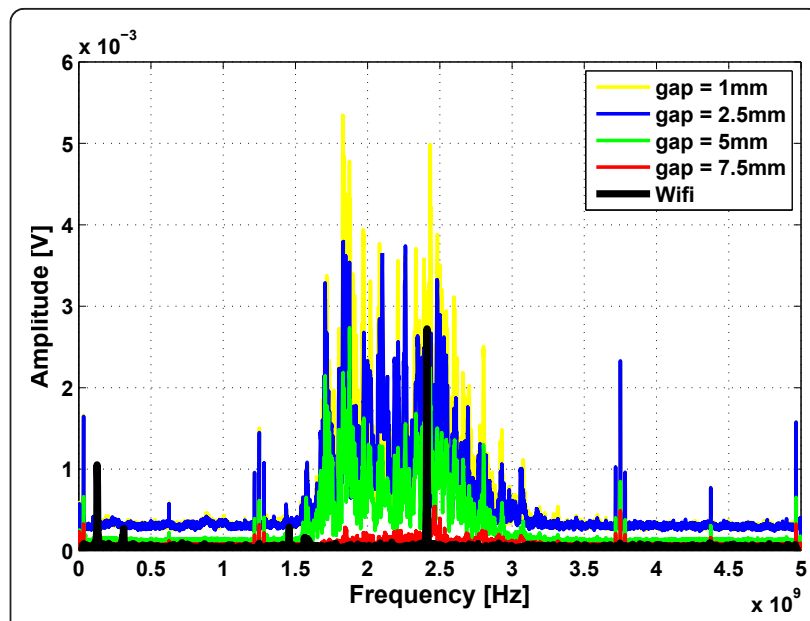

Figure 3 Superimposed spectrums for different gap, Gain $=25$ dB.

data and the extension of Middleton Class A model for multi-antenna system.

\section{A. Middleton Class A model}

Middleton Class A model refers to Narrowband Noise where interference spectrum is narrower than the receiver bandwidth. In this model, the received interference is assumed to be a process having two components $[4,5]$ :

$$
X(t)=X_{P}(t)+X_{G}(t)
$$

where $X_{P}(t)$ and $X_{G}(t)$ are independent processes. They represent the non-Gaussian (impulsive) and Gaussian components, respectively. The probability density function (PDF) of $X(t)$ is given in [4]:

$$
\begin{gathered}
f_{P+G}(x)=e^{-A} \sum_{m=0}^{\infty} \frac{A^{m}}{m ! \sqrt{2 \pi \sigma_{m}^{2}}} e^{\frac{-x^{2}}{2 \sigma_{m}^{2}}} \\
\text { with } \sigma_{m}^{2}=\frac{\frac{m}{A}+\Gamma}{1+\Gamma}
\end{gathered}
$$

Note that $f$ is a weighted sum of zero-mean Gaussians with increasing variance. $A$ and $\Gamma$ are the basic parameters of the model. Let us consider their definitions and physical significance:

1) $A$ is the Overlap Index or Nonstructure Index.

$$
A=v T_{s}
$$

where $v$ is the average number of emission events impinging on the receiver per second and $T_{s}$ is the mean duration of a typical interfering source emission. The smaller $A$ is, the fewer the number of emission (events) and/or their durations. Therefore, the (instantaneous) noise properties are dominated by the waveform 
characteristics of individual events. As $A$ is made larger, the noise becomes less structured, i.e., the statistics of the instantaneous amplitude approach the Gaussian distribution (according to central limit theory [5]). Hence, $A$ is a measure of the non-Gaussian nature of the noise input to the receiver.

2) $\Gamma$ is called the Gaussian factor. It is the ratio of powers in the Gaussian and non-Gaussian components

$$
\Gamma=\frac{\left(X_{G}\right)}{\left(X_{P}\right)}
$$

In general, $A \in\left[10^{-4}, 1\right]$ and $\Gamma \in\left[10^{-6}, 1\right]$ [19]. By adjusting the parameters $A$ and $\Gamma$, the density in (3) can be made to fit a great variety of non-Gaussian noise densities.

\section{B. Validation of Middleton Class A model for partial discharge}

We validated the Middleton Class A model with the measured datasets presented previously by the following procedure of Figure 4. From the measured noise, we used the method of moments [20] to estimate the parameters $A$ and $\Gamma$ of Middleton Class A model:

$$
\begin{gathered}
A_{\text {est }}=\frac{9\left(e_{4}-2 e_{2}^{2}\right)^{3}}{2\left(e_{6}+12 e_{2}^{3}-9 e_{2} e_{4}\right)^{2}} \\
\Gamma_{\text {est }}=\frac{2 e_{2}\left(e_{6}+12 e_{2}^{3}-9 e_{2} e_{4}\right)}{3\left(e_{4}-2 e_{2}^{2}\right)^{3}}
\end{gathered}
$$

where $e_{2}, e_{4}$, and $e_{6}$ are the second, the fourth, and the sixth order moments of the envelope data respectively. These estimated parameters will then be used to generate the noise. In the procedure for validation, three statistical methods are used to compare measured and simulated noises:

1) The probability density function (PDF) is estimated from measured data by using kernel density estimators [21].

2) The complementary cumulative distribution function $(\mathrm{CCDF})$ gives the probability that the random variable is above a particular level and is defined as:

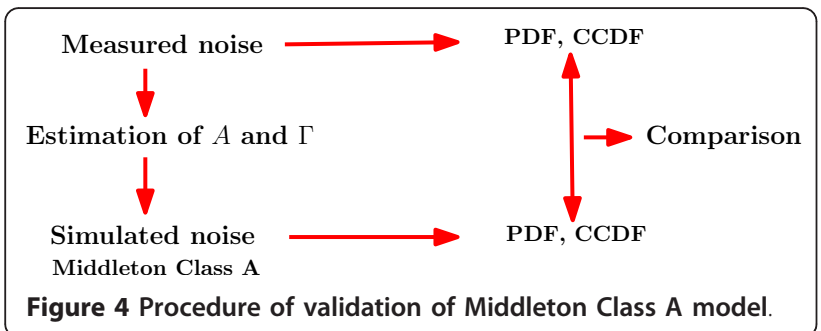

$$
\operatorname{CCDF}(X)=P(X>x)=\int_{x}^{\infty} \operatorname{PDF}(u) \mathrm{d} u=1-\operatorname{CDE}(x)
$$

where $\mathrm{CDF}$ is the cumulative distribution function.

3) The Kullback-Leibler divergence (K-L) is a relative entropic criterion, and it measures the dissimilarity between two probability distributions $P$ and $Q$, where $(\mathrm{K}-\mathrm{L})=0$ indicates that $P=Q[22,23]$.

Figures 5 and 6 show both PDF and CCDF for two measured noises (generator bar and Tesla-Coil), respectively. The estimated parameters for the two measured noises are $\left(A_{\text {est }}=0.0280, \Gamma_{\text {est }}=0.3978\right)$ for generator bar and $\left(A_{\text {est }}=0.3575, \Gamma_{\text {est }}=0.1194\right)$ for Tesla-Coil. We denote Middleton-1 and Middleton- 2 the estimated Middleton class A noise calculated using the estimated parameters $\left(A_{\text {est }}=0.0280, \Gamma_{\text {est }}=0.3978\right)$ and $\left(A_{\text {est }}=\right.$ $\left.0.3575, \Gamma_{\text {est }}=0.1194\right)$, respectively. The PDF and CCDF of the estimated Middleton class A noises and the Gaussian noise are also presented on Figures 5 and 6 . These figures show that the PDF and CCDF of the estimated Middleton class A noises (Middleton-1 and Middleton2) are more close to the measured noises than the Gaussian case. Table 1 confirms these observations by presenting the K-L divergences of the measured noises and the two models of noise (Middleton and Gaussian). So, the K-L divergence of Measured noise- 1 density is 0.04 . from the estimated Middleton Class A density and 0.3 from the Gaussian density. For Measured noise-2 density, the K-L divergence is 0.02 from the estimated Middleton Class A density and 0.27 from the Gaussian density. These results confirm that the measured impulsive noise is better modeled by the Middleton Class A model as compared to Gaussian noise. Hence, we can use the Middleton Class A as an approximated model for impulsive noise in high-voltage substation. Therefore, we evaluate the performance of wireless communication in this environment using the estimated parameters of the measured noises.

\section{Extension of Middleton Class A model for multi- antenna systems}

In order to evaluate the performances of MIMO systems under the impulsive noise, an extension of the Middleton model is derived. Middleton Class A model was derived for single-antenna systems. For a two-antenna system, we considered a bivariate Middleton Class A model used in [7]. This model is limited to $n_{r}=2$ antennas. Thus, we derive an extension for $n_{r} \geq 2$. We can write (3) as:

$$
f(x)=\sum_{m=0}^{\infty} a_{m} g\left(x, \mu, \sigma_{m}^{2}\right)
$$



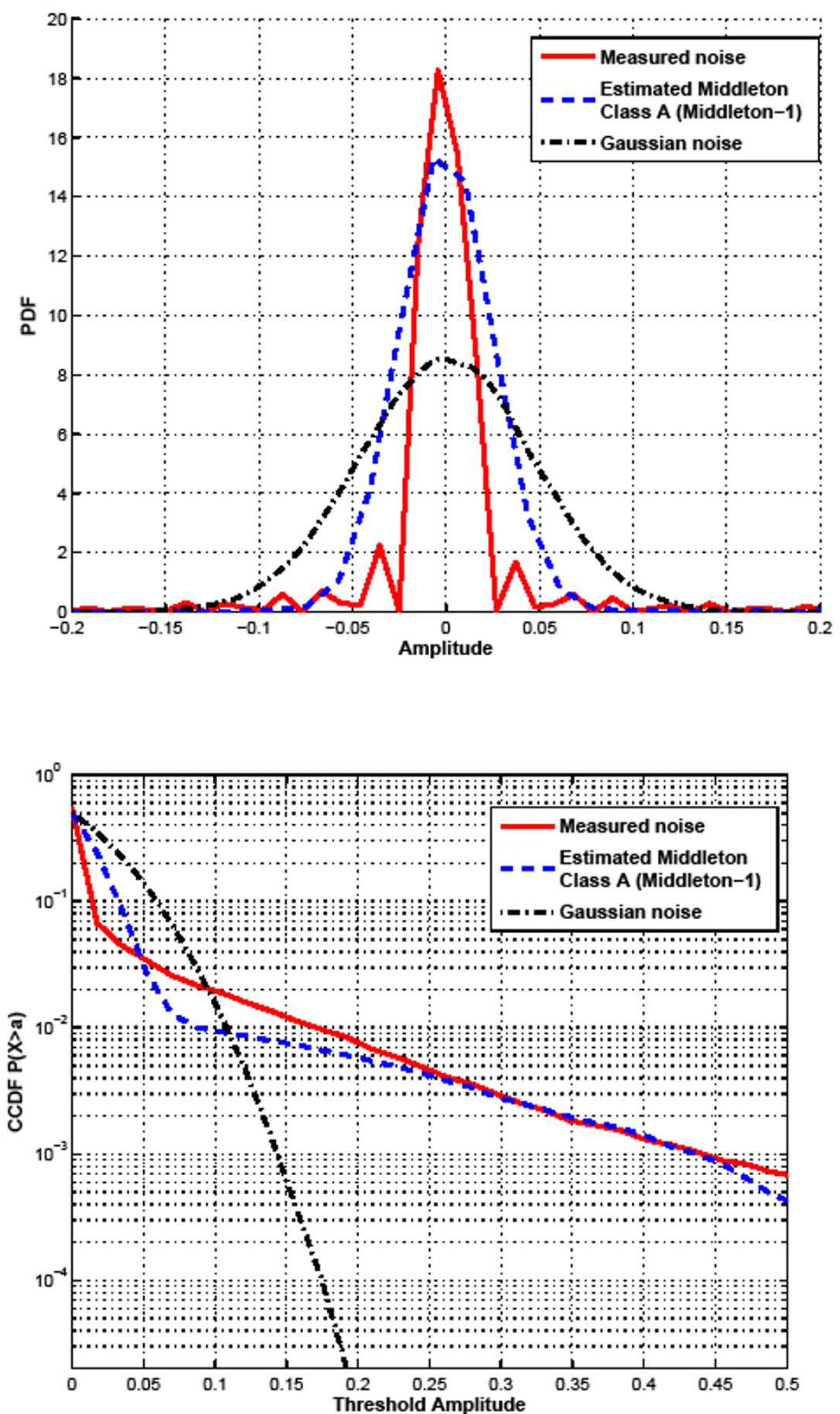

Figure 5 Measured noise-1 (generator bar) PDF and CCDF.

where

$$
a_{m}=\frac{e^{-A} A^{m}}{m !}, \mu=0
$$

and

$g\left(x, \sigma_{m}^{2}\right)=\frac{1}{\sqrt{2 \pi \sigma_{m}^{2}}} e^{\frac{-x^{2}}{2 \sigma_{m}^{2}}}$. The density of Middleton
Class A can be approximated by the two-term model [19]:

$$
f(x)=e^{-A} g\left(x, \sigma_{0}^{2}\right)+\left(1-e^{-A}\right) g\left(x, \sigma_{1}^{2}\right)
$$



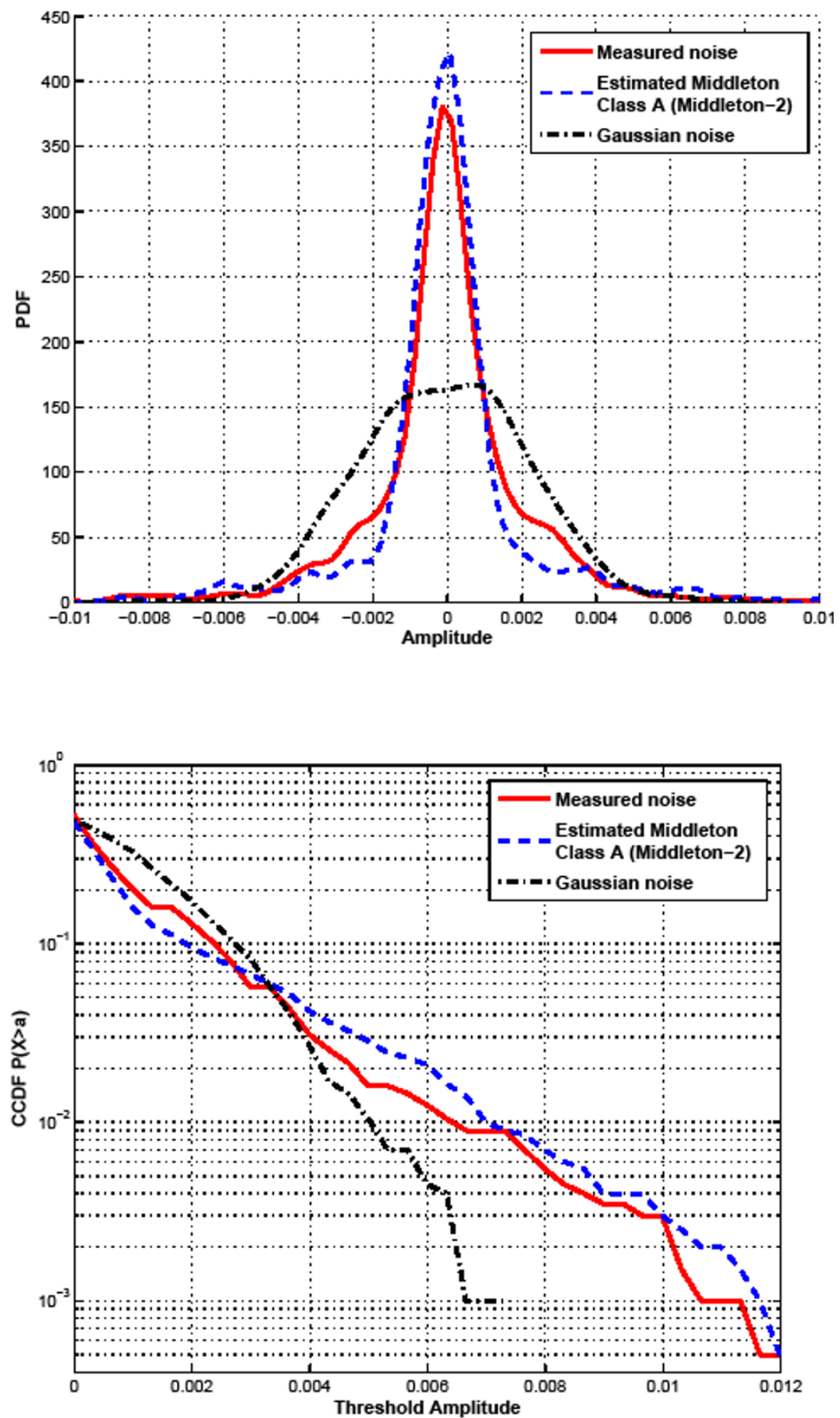

Figure 6 Measured noise-2 (Tesla-Coil) PDF and CCDF.

Table 1 K-L Divergences

\begin{tabular}{lll}
\hline & Measured noise-1 & Measured noise-2 \\
\hline Class A & 0.04 & 0.02 \\
\hline Gaussian & 0.3 & 0.27 \\
\hline
\end{tabular}

Let $\mathrm{x}=\left[x_{1}, x_{2}, x_{3}, \ldots, x_{k}\right]$ be a vector of $k=n_{r}$ random variables, each variable has a Middleton Class A density function and $x_{k}$ is the noise observation at the $k$ th antenna. Then, the multivariate density of $\mathrm{x}$ can be 
written as [19]:

$$
f_{x}(x)=\sum_{m=0}^{\infty} a_{m} g\left(\mathrm{x}, K_{m}\right)
$$

where $a_{m}$ is as in (9), $K_{m}$ is the covariance matrix that represents the spatial correlation in the noise and $g$ is a multivariate Gaussian function:

$$
g\left(\mathrm{x}, K_{m}\right)=\frac{1}{(2 \pi)^{\frac{n_{r}}{2}}\left|K_{m}\right|^{\frac{1}{2}}} e^{\frac{-\mathrm{x}^{T} K_{m}^{-1} \mathrm{x}}{2}}
$$

where $|$.$| denotes the determinant. From (11) and (12),$ we obtain:

$$
f_{x}(x)=\sum_{m=0}^{\infty} \frac{a_{m}}{(2 \pi)^{\frac{n_{r}}{2}}\left|K_{m}\right|^{\frac{1}{2}}} e^{\frac{-\mathrm{x}^{T} K_{m}^{-1} \mathrm{x}}{2}}
$$

Equation (13) represents a general extension of Middleton Class A model for multi-antenna systems. We can use the approximation as in (10). Then, we obtain an approximate version of the extension:

$$
\begin{aligned}
f_{x}(x)= & \frac{e^{-A}}{(2 \pi)^{\frac{n_{r}}{2}}\left|K_{0}\right|^{\frac{1}{2}}} e^{\frac{-\mathrm{x}^{T} K_{0}^{-1} \mathrm{x}}{2}} \\
& +\frac{1-e^{-A}}{(2 \pi)^{\frac{n_{r}}{2}}\left|K_{1}\right|^{\frac{1}{2}}} e^{\frac{-\mathrm{x}^{T} K_{1}^{-1} \mathrm{x}}{2}}
\end{aligned}
$$

where $K_{m}$ is $n_{r} \times n_{r}$ covariance matrix and is defined as:

$$
\begin{gathered}
K_{m}=\left(\begin{array}{ccc}
\operatorname{Var}\left(x_{1}\right)_{m} & \ldots & \operatorname{Cov}\left(x_{1}, x_{k}\right)_{m} \\
\vdots & \ddots & \vdots \\
\operatorname{Cov}\left(x_{k}, x_{1}\right)_{m} & \ldots & \operatorname{Var}\left(x_{k}\right)_{m}
\end{array}\right) \\
\text { where }\left\{\begin{array}{l}
\operatorname{Var}\left(x_{k}\right)_{m}=\frac{\frac{m}{A}+\Gamma_{k}}{1+\Gamma_{k}}=\sigma_{k m}^{2} . \\
\operatorname{Cov}\left(x_{i}, x_{j}\right)_{m}=\rho_{i j} \sigma_{i m} \sigma_{j m}
\end{array}\right.
\end{gathered}
$$

$\Gamma_{k}$ is the Gaussian factor at the $k$ th antenna and $\rho_{i j}$ is the correlation coefficient between the noise observations at $i$ and $j$ antennas, $-1 \leq \rho \leq 1$. Finally, we can write $K_{m}$ as

$$
K_{m}=\left(\begin{array}{ccc}
\sigma_{1 m}^{2} & \ldots & \rho_{1 k} \sigma_{1 m} \sigma_{k m} \\
\vdots & \ddots & \vdots \\
\rho_{k 1} \sigma_{k m} \sigma_{1 m} & \cdots & \sigma_{k m}^{2}
\end{array}\right)
$$

\section{MIMO systems used in simulation}

\section{A. MIMO precoders}

As mentioned in the introduction, the MIMO system used is based on precoding with the assumption that the CSI is available at both transmit and receive sides. The use of CSI allows designing precoders by optimizing a pertinent criteria as maximizing the received signal-to-noise ratio (max-SNR or beamforming), minimizing the mean square error (MMSE), maximizing the capacity (Water-Filling solution) [24], or the maximization of the minimum Euclidean distance of received constellation ( $\max -d_{\min }$ solution) [11]. All these linear precoders are based on the singular value decomposition (SVD) of the channel matrix. The max $-d_{\text {min }}$ precoder achieves good performances in terms of BER providing a significant gain of SNR compared to the other precoders (max-SNR, MMSE, and Water-Filling) [25], and it will be used in our MIMO system.

Let us consider a MIMO system with $n_{t}$ transmit and $n_{r}$ receive antennas over which we want to achieve $b$ independent data streams $\left(b \leq \min \left(n_{t}, n_{r}\right)\right)$. The received signal can then be expressed as:

$$
\mathrm{y}=\mathrm{GHFs}+\mathbf{G v}
$$

where $\mathbf{y}$ is the $b \times 1$ received vector, $\mathbf{s}$ is the $b \times 1$ symbols vector of the constellation $C, \mathbf{v}$ is an additive noise vector of size $n_{r} \times 1, \mathbf{H}$ is the channel matrix, and $\mathbf{F}$ and $\mathbf{G}$ are the precoder and decoder matrices, respectively. In our case, the additive noise is the Middleton Class A model.

\section{B. Presentation of selected precoders}

The precoder is presented using a virtual transformation of the system in (17) [11]. By considering the following decompositions $\mathbf{F}=\mathbf{F}_{v} \mathbf{F}_{d}$ and $\mathbf{G}=\mathbf{G}_{d} \mathbf{G}_{v}$, the input-output relation (17) can be re-expressed as:

$$
\mathbf{y}=\mathbf{G}_{d} \mathbf{H}_{v} \mathbf{F}_{d} \mathbf{s}+\mathbf{G}_{d} \mathbf{v}_{v}
$$

This procedure is frequently used for MIMO linear precoder systems, and it is based on the SVD of the channel matrix $\mathbf{H}[11,24]$. The matrix $\mathbf{H}_{v}=\mathbf{G}_{v} \mathbf{H F}_{v}=$ $\operatorname{diag}\left(\sigma_{1}, \ldots, \sigma_{b}\right)$ is the virtual channel matrix of size $b \times$ $b, \sigma_{i}$ stands for every subchannel gain (sorted by decreasing order), $\mathbf{v}_{v}=\mathbf{G}_{v} \mathbf{v}$ is the virtual noise, $\mathbf{G}_{v}$ and $\mathbf{F}_{v}$ are unitary matrices obtained from applying the SVD operation on the channel matrix. $\mathbf{F}_{d}$ and $\mathbf{G}_{d}$ are $b \times b$ matrices, representing the precoder and decoder in the virtual channel. The power constraint is expressed as $\operatorname{trace}\{\mathbf{F F} *\}=\operatorname{trace}\left\{\mathbf{F}_{d} \mathbf{F}_{d}^{*}\right\}=p_{0}$, where $p_{0}$ is the mean available transmit power. As only ML detection is 
considered in the rest of the paper, the decoder matrix $\mathbf{G}_{d}$ has no impact on the performance and is consequently assumed to be equal to $\mathbf{I}_{b}$, the identity matrix of size $b \times b$ [11].

The max $-d_{\text {min }}$ precoder maximizes the minimum Euclidean distance between signal points at the receiver side $d_{\text {min }}$ which is defined by:

$$
d_{\min }\left(\mathbf{F}_{d}\right)=\min _{\left(\mathbf{s}_{k}, \mathbf{s}_{l}\right) \in C^{b}, \mathbf{s}_{k} \neq \mathbf{s}_{l}}\left\|\mathbf{H}_{v} \mathbf{F}_{d}\left(\mathbf{s}_{k}-\mathbf{s}_{l}\right)\right\|
$$

where $\mathbf{s}_{k}$ and $\mathbf{s}_{l}$ are 2 symbol vectors whose entries are elements of $C$. Then, the max- $d_{\text {min }}$ precoder is the solution of:

$$
\mathbf{F}_{d}=\underset{\mathbf{F}_{d}}{\arg \max } d_{\min }\left(\mathbf{F}_{d}\right)
$$

A very exploitable solution of (20) is given in [11] for two independent data streams, $b=2$ and a 4-QAM with a spectral efficiency of $4 \mathrm{bit} / \mathrm{s} / \mathrm{Hz}$. Recently, the solution with two 16-QAM symbols was also given [26]. This extension permits to increase the spectral efficiency to 8 $\mathrm{bit} / \mathrm{s} / \mathrm{Hz}$. The max-SNR precoder consists in maximizing the SNR and transmitting one single symbol. The modulation is chosen in order to maintain the same spectral efficiency.

We will also use another linear precoding system proposed in [27] named ARITH-BER precoder. This precoder minimizes the average BER or the arithmetic mean of the BER (ARITH-BER). It is based on the Schur-convex optimization.

There are other precoding design studied in the literature, for example, the P-OSM precoder. This precoder uses a new orthogonal spatial multiplexing (OSM) scheme transmitting two independent data streams $(b=$ 2) $[12,28]$. The P-OSM precoder maximizes the minimum distance like the max- $d_{\min }$ but it is not based on the SVD operation. It simplifies the ML detection by searching for a single symbol (called single symbol decodable), while the ML in max- $d_{\min }$ requires searching a pair of symbols. However, the P-OSM precoder assumes $n_{t}=2$ transmit antennas and when $n_{t}>2$, it should be associated with an antenna-selection scheme.

\section{Simulation results}

The performance of MIMO precoders presented in Section IV is evaluated in terms of BER in the presence of impulsive noise. The parameters $A$ and $\Gamma$ estimated in Section III-B were used to generate the corresponding noise. In measurement setup, single antenna is used to capture the impulsive noise, and we do not have yet measures for $n_{r}$ receive multi-antenna configuration. In the noise model for multi-antenna system presented in III-C, we need the Gaussian factor $\Gamma$ at each receive antenna and the correlation coefficient $\rho$ between the noise observations at receive antennas. These two parameters can be estimated from measuring the noise in a multi-antenna configuration. Therefore, in order to launch simulation with the noise model for multiantenna system, we assume a simple case: $\left(\Gamma_{\text {est }}=\Gamma_{1}=\Gamma_{2}=\cdots=\Gamma_{n_{r}}\right)$ and there is no correlation between noise observations at antennas $(-1 \leq \rho \leq 1$, we put $\left.\rho_{i j}=\rho_{j i}=0\right)$. The Middleton Class A model is defined for only real sample observation. For complex signals (QAM modulation), we assume that the real and the imaginary parts of the signal are independent and identically distributed (i.i.d). A flat Rayleigh-fading channel was used, i.e., $\mathbf{H}$ is an $\left(n_{r} \times n_{t}\right)$ channel matrix with independent and identical distributed complex Gaussian entries with mean zero and unit variance. We simulated the MIMO precoders in several cases: with 4-QAM or 16-QAM, $(2 \times 2)$, $(2$ $\times 4)$ or $(4 \times 4)$ MIMO systems. $b$ is always equal to 2 symbols in max- $d_{\min }$, ARITH-BER and P-OSM precoders, while it is equal to 1 in max-SNR precoder.

We consider an ML MIMO receiver. MIMO receivers have been typically designed under the assumption of additive white Gaussian noise. In our work, the wireless system is envisaged to work in electricity substations where the impulsive noise is prevalent. Thus, we first evaluate the performances of MIMO systems in the presence of impulsive noise using the ML receiver with Gaussian noise assumption (ML-GN). Next, we apply an ML receiver with the assumption of impulsive noise (ML-IN). The goal is to see whether we can get an important improvement in performance between the two cases which could justify the use of such a receiver since its application is more complex than the ML-GN one as we will see later.

\section{A. ML under Gaussian noise assumption: ML-GN}

Figure 7 shows a degradation of BER of the max $-d_{\text {min }}$ precoder $(2 \times 2$ and $2 \times 4 \mathrm{MIMO})$ in the presence of impulsive noise (Middleton-1 and Middleton-2). The energy of the Middleton Class A model is a sum of two components of noise (Gaussian and impulsive). At low SNR, the BER is sensitive to the Gaussian component of the Middleton Class A noise, which has lower energy than a classical Gaussian noise. Hence, BER of Middleton Class A is better compared to classical Gaussian noise at low SNR. At high SNR, the MIMO system becomes sensitive to the impulsive component, and this degrades the performance of the wireless systems in EMI (SNR loss can reach $5 \mathrm{~dB}$ ). The BER of Middleton1 noise is more degraded than Middleton- 2 case since the parameter $A$ of Middleton-1 is smaller than that of Middleton-2. When the value of $A_{\text {est }}$ increases, the Gaussian component increases and the BER of Middleton-2 is close to the Gaussian case. Moreover, Figure 7 


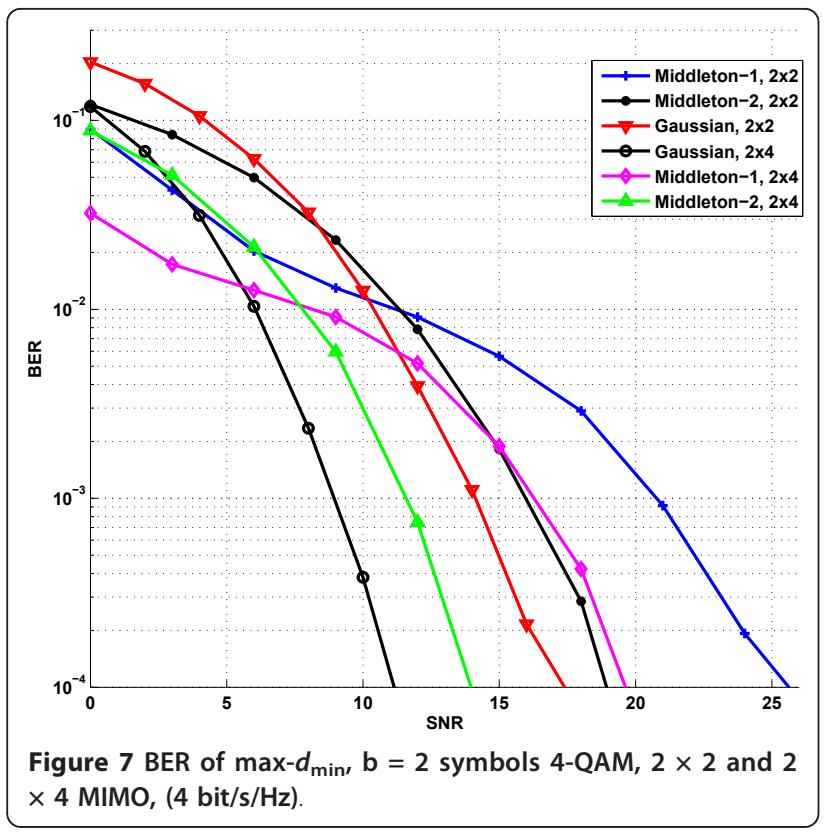

also shows the influence of the number of receive antennas. When we increased $n_{r}$ from 2 to 4 , the BER is improved with a SNR gain near $4 \mathrm{~dB}$. We can also observe that the impulsive noise influences the diversity order. Indeed, the max- $d_{\min }$ precoder achieves the maximum diversity order $n_{t} \times n_{r}$ in the Gaussian case. In the Middleton case, the diversity is lower.

For $2 \times 2$ MIMO system and a perfect or imperfect CSI, we showed in [29] that the max- $d_{\min } 16-\mathrm{QAM}$ precoder achieved a better BER than the max-SNR (256QAM) one with a spectral efficiency of $8 \mathrm{bit} / \mathrm{s} / \mathrm{Hz}$ and for Gaussian noise. This performance of $\max -d_{\min } 16-$ QAM is also similar for $4 \times 4$ MIMO and Gaussian noise. Hence, we evaluated the performance of these precoders with $4 \times 4$ MIMO system, and in the presence of impulsive noise, the performance of P-OSM and ARITH-BER is also evaluated. Figure 8 shows the BER for max- $d_{\text {min }}$, max-SNR, P-OSM and ARITH-BER precoders for only Middleton-1. The max- $d_{\min }$ is still better than the max-SNR. The BER of max- $d_{\text {min }}$ is smaller than the BER of P-OSM at low and high SNR. The ARITH-BER seems to have a slight improvement compared with $\max -d_{\min }$ precoder. However, for a certain SNR (15 dB), the BERs of all precoders are close. It means that they are sensitive to the transition of the impulsive noise with a particular SNR.

\section{B. ML under impulsive noise assumption: ML-IN}

The detection rule for the ML receiver is given as

$$
\hat{\mathbf{s}}=\underset{s \in C}{\arg \max }\{\mathbf{L}(\mathbf{s} \mid \mathbf{y})\}
$$

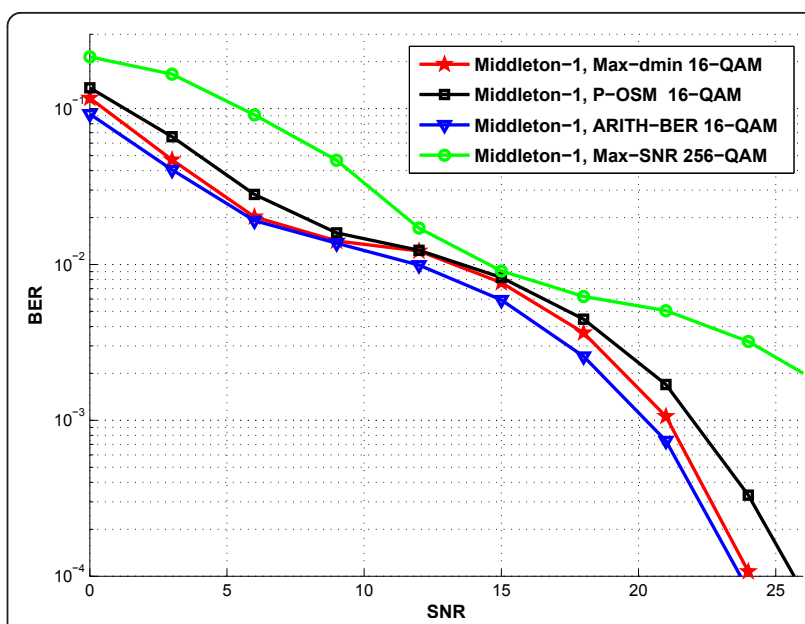

Figure 8 BER of $\max -d_{\min }$, P-OSM, ARITH-BER, $b=2$ symbols 16-QAM and max-SNR $b=1$ symbol 256-QAM, $4 \times 4$ MIMO, (8 $\mathrm{bit} / \mathrm{s} / \mathrm{Hz}$ ).

In Gaussian noise, the likelihood function is expressed as the distance between the received signal and candidate points in the constellation (distance metric). In Middleton Class A noise, this distance can not be attained. For this ML receiver, we use the probability density function of the Middleton noise model for multi-antenna systems given in (14). In this case, The ML detection can be expressed as the maximizing of the likelihood function $\mathbf{L}(\mathbf{s} / \mathbf{y})$

$$
\begin{aligned}
\mathbf{L}(\mathbf{s} \mid \mathbf{y})= & \left\{\frac{e^{-A}}{(2 \pi)^{\frac{n_{r}}{2}}\left|\mathrm{~K}_{0}\right|^{\frac{1}{2}}} e^{\frac{-\mathrm{x}^{T} K_{0}^{-1} \mathrm{x}}{2}}\right. \\
& \left.+\frac{1-e^{-A}}{(2 \pi)^{\frac{n_{r}}{2}}\left|\mathrm{~K}_{1}\right|^{\frac{1}{2}}} e^{\frac{-\mathrm{x}^{T} K_{1}^{-1} \mathrm{x}}{2}}\right\}
\end{aligned}
$$

where $\mathbf{x}=\mathbf{y}$ - HFs, $\left[n_{r} \times 1\right]$

Figure 9 shows the BER of max- $d_{\min }$, P-OSM, and ARITH-BER precoders with the ML-IN receiver (under impulsive noise assumption). The performance of this ML-IN receiver is significantly better (gain of $7 \mathrm{~dB}$ ) than the ML-GN one. However, in case of P-OSM precoder, the ML-IN receiver is no longer single symbol decodable. It now searches a pair of symbols like the max$d_{\text {min }}$, and ARITH-BER because the ML-IN receiver is only possible for a joint $\mathrm{ML}$ detection.

The ML-IN receiver seemed to present an important gain of SNR (7 dB) compared to ML-GN receiver. This gain may be very useful in a cooperative MIMO for WSN in substation environments. Since the reduction of SNR ratio while still ensuring the same target BER leads to save the power consumption in WSN by 


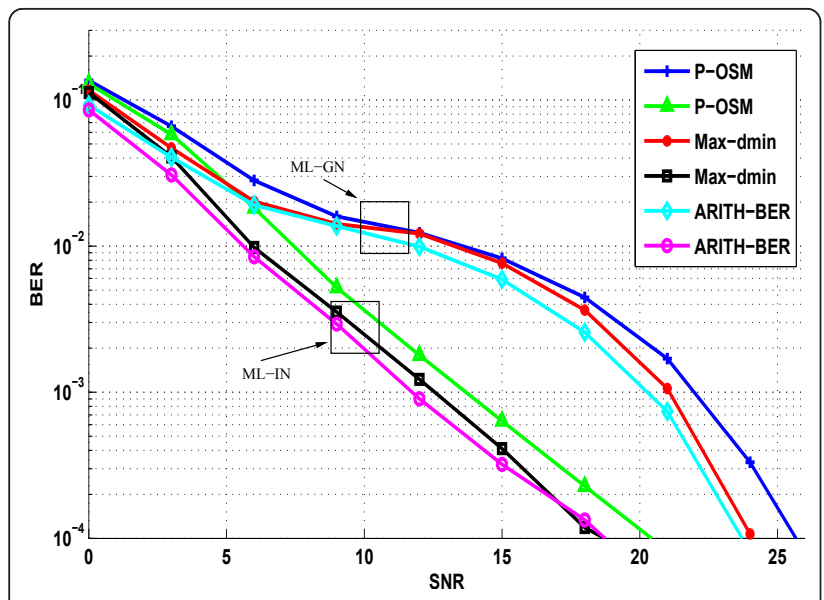

Figure 9 BER of $\max -d_{\min }, \mathrm{P}-\mathrm{OSM}$ and ARITH-BER, $b=2$ symbols $4 \times 4$ MIMO, 16-QAM, (8 bit $/ \mathrm{s} / \mathrm{Hz})$, Middleton-1 noise.

reducing the transmission energy [9]. However, the computational complexity of this receiver is higher than its ML-GN counterpart. Furthermore, it requires the knowledge of the noise model parameters (A and $\Gamma$ ). Therefore, this receiver should be associated with an estimator of noise parameters, or the electromagnetic environment should be heavily studied, and tests in laboratories and in sites should be performed in order to characterize the noise and its parameters.

\section{Conclusion}

The works presented deal with the modeling of an impulsive noise in a high-voltage substation and its impacts on promising MIMO techniques such as linear precoding. The specimens used in this study reveal that the methodology for measuring impulsive electromagnetic noise is valid for any kind of air partial discharges. The applied voltage and the physical dimensions (gap) have a direct influence on the impulsive noise spectrum: a high voltage and a small gap can move the average frequency to the high frequencies (above $1 \mathrm{GHz}$ ). Moreover, the statistical modeling of the measurements showed that Middleton Class A can be used as an approximated model for impulsive noise in high-voltage substations. Using the estimated parameters of the measured noise and the MIMO extension of the Middleton model, we have evaluated the performance of several MIMO precoders with channel state information at both transmitter and receiver. At a high SNR, the performance of precoders was degraded in the presence of impulsive noise compared to Gaussian one. The ML-IN receiver, i.e. under an impulsive noise assumption, seemed to present an important gain $(7 \mathrm{~dB})$, but the complexity is increased. For future works, we recommend to do some additional measurements with a higher voltage (up to $200 \mathrm{kV}$ in laboratory and more in substations) and extend the focus around ISM bands to other wireless technologies like ZigBee, Wimax, or LTE. It will also be interesting to validate the multi-antenna extension model with measured data noise using $n_{r}$ antennas in measurement setup as in a MIMO configuration.

\section{Acknowledgements}

This work has been done in a scientific collaboration between XLIM-SIC laboratory (France), Hydro-Québec's Research Institute, and École de technologie supérieure (Canada) as part of project for wireless sensors communication in disturbed environments. It was supported by the Samuel De Champlain program between France and Québec.

\section{Author details}

${ }^{1}$ XLIM-SIC UMR CNRS 6172 Bvd., Marie et Pierre Curie, 86962 Futuroscope Cedex, France ${ }^{2}$ Institut de Recherche d'Hydro-Québec, 1800 Lionel-Boulet, Varennes, QC, J3X-1S1, Canada ${ }^{3}$ École de technologie superieure, 1100 Notre-Dame ouest, Montréal, QC, H3C-1K3, Canada

\section{Competing interests}

The authors declare that they have no competing interests.

Received: 26 February 2011 Accepted: 25 November 2011 Published: 25 November 2011

\section{References}

1. GW Arnold, Challenges and opportunities in smart grid: a position article. Proc IEEE. 99(6), 922-927 (2011)

2. W Pakala, V Chartier, Radio noise measurements on overhead power lines from 2,4 to $800 \mathrm{kv}$. IEEE Trans Power Appl Syst. PAS-90, 1155-1165 (1971)

3. W Pakala, E Taylor, R Harrold, Radio noise measurements on high voltage lines from 2.4 to 345 kv. IEEE Trans Electromagn Compat. 10, 96-107 (1968)

4. A Spaulding, D Middleton, Optimum reception in an impulsive interference environment-part 1: coherent detection. IEEE Trans Commun, 9: 910-923 (1977)

5. D Middleton, Non-gaussian noise models in signal processing for telecommunications: new methods and results for class A and class B noise models. IEEE Trans Inf Theory. 45(4), 1129-1149 (1999). doi:10.1109/ 18.761256

6. S Miyamoto, M Katayama, N Morinaga, Performance analysis of QAM systems under class A impulsive noise environment. IEEE Trans Electromagn Compat. 37(2), 260-267 (1995). doi:10.1109/15.385891

7. K Gulati, A Chopra, R Heath, B Evans, K Tinsley, X Lin, MIMO receiver design in the presence of radio frequency interference, in IEEE Globecom (2008)

8. S Cui, AJ Goldsmith, A Bahai, Energy-efficiency of MIMO and cooperative MIMO techniques in sensor networks. IEEE J Sel Areas Commun. 22(6), 1089-1098 (2004). doi:10.1109/JSAC.2004.830916

9. SK Jayaweera, Virtual MIMO-based cooperative communication for energyconstrained wireless sensor networks. IEEE Trans Wireless Commun. 5(5), 984-989 (2006)

10. M Islam, K Jinsang, On the cooperative MIMO communication for energyefficient cluster-to-cluster transmission at wireless sensor network, in Ann Telecommun, vol. 65. (Springer, Paris, 2010), pp. 325-340. doi:10.1007/ s12243-009-0151-9

11. L Collin, O Berder, P Rostaing, G Burel, Optimal minimum distance-based precoder for MIMO spatial multiplexing systems. IEEE Trans Signal Process. 52(3), 617-627 (2004). doi:10.1109/TSP.2003.822365

12. Y Kim, H Lee, S Park, I Lee, Optimal precoding for orthogonalized spatial multiplexing in closed-loop MIMO systems. IEEE J Sel Areas Commun. 26(8), 1556-1566 (2008)

13. TN Zogakis, PS Chow, JT Aslanis, JM Cioffi, Impulse noise mitigation strategies for multicarrier modulation, in Proc IEEE Int Conf Commun, vol. 2. Geneva, pp. 784-788 (1993)

14. T Faber, T Scholand, P Jung, On turbo codes for environments impaired by impulsive noise, in Vehicular Technology Conference, VTC2004-Fall (2004) 
15. FH Gregorio, JE Cousseau, JL Figueroa, Reducing impulsive noise in DSL systems -robustness and delay, in Proceedings of the 6th Nordic Signal Processing Symposium-NORSIG (2004)

16. http://www.electrotechnicproduct.com/pinhole.asp

17. I Moore, I Portuguks, A Glover, A non-intrusive partial discharge measurement system based on RF technology, in Power Engineering Society General Meeting, IEEE (2003)

18. T Babnik, R Aggarwal, PJ Moore, Z Wang, Radio frequency measurement of different discharges. in IEEE Bologna PowerTech Conference 23-26 (June 2003)

19. PA Delaney, Signal detection in multivariate class A interference. IEEE Trans Commun. 43(2), 365-373 (1995). doi:10.1109/26.380055

20. D Middleton, Procedures for determining the properties of the first-order canonical models of class A and class B electromagnetic interference. IEEE Trans Electromagn Compat. 21, 190-208 (1979)

21. Zl Botev, A novel nonparametric density estimator. The University of Queensland, Tech Rep (2006)

22. S Kullback, Information Theory and Statistics (Peter Smith, Gloucester, 1978)

23. M Basseville, Information: entropies, divergences and mean values. IRISA 1020, Rennes France: Institute of Research in Computer Sciences and Random Systems. (1996)

24. H Sampath, P Stoica, A Paulraj, Generalized linear precoder and decoder design for MIMO channels using the weighted MMSE criterion. IEEE Trans Commun. 49(12), 2198-2206 (2001). doi:10.1109/26.974266

25. B Vrigneau, J Letessier, P Rostaing, L Collin, G Burel, Statistical comparison between max-dmin, max-SNR and MMSE precoders, in 40th Asilomar Conference on Signals, Systems and Computers (2006)

26. Quoc-tuong, O Berder, B Vrigneau, O Sentieys, Minimum distance based precoder for MIMO-OFDM systems using a 16-QAM modulation, IEEE Int Conf Commun ICC 1-5, (Germany, 2009)

27. D Palomar, J Cioffi, M Lagunas, Joint tx-rx beamforming design for multicarrier MIMO channels: a unified framework for convex optimization. Signal Process IEEE Trans. 51(9), 2381-2401 (2003). doi:10.1109/ TSP.2003.815393

28. H Lee, S Park, I Lee, Orthogonalized spatial multiplexing for closed-loop MIMO systems. IEEE Trans Commun. 55, 1044-1052 (2007)

29. G Madi, B Vrigneau, Y Pousset, R Vauzelle, A realistic MIMO time-variant channel applied to diagonalizing precoders, in The 9th International Conference on ITS Telecommunications, (France, 2009)

doi:10.1186/1687-1499-2011-186

Cite this article as: Madi et al:: Impacts of impulsive noise from partial discharges on wireless systems performance: application to MIMO precoders. EURASIP Journal on Wireless Communications and Networking 2011 2011:186.

\section{Submit your manuscript to a SpringerOpen ${ }^{\circ}$ journal and benefit from:}

- Convenient online submission

- Rigorous peer review

- Immediate publication on acceptance

- Open access: articles freely available online

- High visibility within the field

- Retaining the copyright to your article

Submit your next manuscript at $\gg$ springeropen.com 